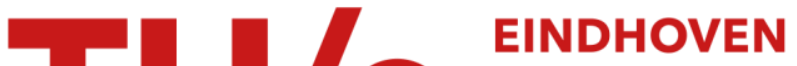

\section{Overview of analytical models for the design of linear and planar motors}

Citation for published version (APA):

Jansen, J. W., Smeets, J. P. C., Overboom, T. T., Rovers, J. M. M., \& Lomonova, E. A. (2014). Overview of analytical models for the design of linear and planar motors. IEEE Transactions on Magnetics, 50(11), 8206207-. https://doi.org/10.1109/TMAG.2014.2328556

DOI:

10.1109/TMAG.2014.2328556

Document status and date:

Published: 01/01/2014

\section{Document Version:}

Publisher's PDF, also known as Version of Record (includes final page, issue and volume numbers)

\section{Please check the document version of this publication:}

- A submitted manuscript is the version of the article upon submission and before peer-review. There can be important differences between the submitted version and the official published version of record. People interested in the research are advised to contact the author for the final version of the publication, or visit the $\mathrm{DOI}$ to the publisher's website.

- The final author version and the galley proof are versions of the publication after peer review.

- The final published version features the final layout of the paper including the volume, issue and page numbers.

Link to publication

\section{General rights}

Copyright and moral rights for the publications made accessible in the public portal are retained by the authors and/or other copyright owners and it is a condition of accessing publications that users recognise and abide by the legal requirements associated with these rights.

- Users may download and print one copy of any publication from the public portal for the purpose of private study or research.

- You may not further distribute the material or use it for any profit-making activity or commercial gain

- You may freely distribute the URL identifying the publication in the public portal.

If the publication is distributed under the terms of Article 25fa of the Dutch Copyright Act, indicated by the "Taverne" license above, please follow below link for the End User Agreement:

www.tue.nl/taverne

Take down policy

If you believe that this document breaches copyright please contact us at:

openaccess@tue.nl

providing details and we will investigate your claim. 


\title{
Overview of Analytical Models for the Design of Linear and Planar Motors
}

\author{
J. W. Jansen, J. P. C. Smeets, T. T. Overboom, J. M. M. Rovers, and E. A. Lomonova \\ Department of Electrical Engineering, Eindhoven University of Technology, Eindhoven 5612 AZ, The Netherlands
}

\begin{abstract}
In this paper, an overview of analytical techniques for the modeling of linear and planar permanent-magnet motors is given. These models can be used complementary to finite element analysis for fast evaluations of topologies, but they are indispensable for the design of magnetically levitated planar motors and other coreless multi-degrees of freedom motors, which are applied in (ultra) high-precision applications. The analytical methods describe the magnetic fields based on magnetic surface charges and Fourier series in 2-D and 3-D.
\end{abstract}

Index Terms - Analytical models, linear synchronous motors, magnetic levitation, permanent magnets (PM), planar motors.

\section{INTRODUCTION}

$\mathbf{I}^{\mathrm{N}}$ $\mathrm{N}$ high-precision motion systems, linear and planar permanent-magnet (PM) motors are commonly applied in multi-degrees of freedom (DOF) motion systems because of their linearity and low stiffness. To obtain a high-position accuracy during dynamic motion, a good predictability of the force is a prerequisite for these motors. Furthermore, the integration of these motors in light-weight constructions to increase the acceleration results in a further integration of functionalities and the requirement to compensate and control local mechanical deformation in these actuator systems.

In such high-demanding applications, accurate modeling is an essential part in the design and control of linear and planar motors. Finite element analysis (FEA) can be rather complicated and slow due to the large volume in which the energy conversion takes place. Analytical models can be an alternative when they accurately describe all complex boundaries and particularities, such as slotting, end-effects, force distributions, and parasitic force and torque components. The two most developed models are the harmonic model and the surface charge model. Harmonic models are applied to describe magnetic fields in periodical structures [1], [2]. Extensions have been made to include slotting [3], [4] and, recently, to describe systems in the 3-D space [5]. Surface charge models describe the field of separate PMs and can be applied in structures without slotting [6]. As a result, not only the force but also its distribution in a magnet array can be obtained, which cannot be predicted with FEA.

This paper describes these two modeling classes for linear and planar motor structures. Examples of the application of these models are given.

\section{3-D Magnetic Field Modeling}

An analytical expression for the magnetic flux density in linear or planar motors can be derived by applying the static

Manuscript received March 7, 2014; accepted May 24, 2014. Date of current version November 18, 2014. Corresponding author: J. W. Jansen (e-mail: j.w.jansen@tue.nl).

Color versions of one or more of the figures in this paper are available online at http://ieeexplore.iee.org.

Digital Object Identifier 10.1109/TMAG.2014.2328556 field theory in which steady currents and stationary charge distributions are modeled by means of the four coupled firstorder Maxwell equations. The Maxwell equations can be expressed by uncoupled second-order equations by writing them in terms of the magnetic vector and scalar potential, $\mathbf{A}$ and $\Psi$, respectively. These second-order differential equations are written in the form of a Poisson or Laplace equation.

Two different methods can be applied to solve these secondorder differential equations, and, thereby, model the flux density distribution in linear or planar motors. First, the complete magnetization vector or current density distribution can be described with Fourier series. The magnetic fields are obtained by the harmonic modeling technique, in which the method of separations of variables is applied to derive a solution for the Poisson or Laplace equation. Second, each magnet or coil can be individually modeled by solving the second-order differential equations by means of the free-space Green's function, which leads to the current or the charge model for the magnetic vector and magnetic scalar potential, respectively.

\section{A. Harmonic Modeling}

For periodical structures, the harmonic modeling is a suitable method to describe the magnetic flux density. The structure may contain complex iron boundaries, such as slots. In this method, the geometry is subdivided into regions. These volumes deviate from each other due to different material properties and the presence of magnets or coils [5]. A region is defined to be continuous if the length of the region is equal to the pitch of the periodicity of the motor, and, is referred to as non-continuous if the pitch of the region is smaller than the periodicity. Non-continuous regions are, for example, a slot or a cavity, as shown in Fig. 1. In case of linear or planar machines, both the magnets and coils may be considered, and, therefore, the harmonic model is presented in terms of the magnetic vector potential.

The magnetic flux density distribution, $\mathbf{B}$, can be written in terms of the magnetic vector potential as

$$
\mathbf{B}=\nabla \times \mathbf{A} .
$$

0018-9464 (C) 2014 IEEE. Personal use is permitted, but republication/redistribution requires IEEE permission.

See http://www.ieee.org/publications_standards/publications/rights/index.html for more information. 
The magnetization vector, $\mathbf{M}$, is defined as

$$
\begin{aligned}
& \mathbf{M}=\chi \mathbf{H}+\mathbf{M}_{0} \\
& \mathbf{M}=\frac{\mathbf{B}_{r}}{\mu_{0}}
\end{aligned}
$$

where $\chi$ is the magnetic susceptibility, $\mathbf{M}_{0}$ is the residual magnetization and $B_{r}$ is the remanent flux density. This definition of the magnetization vector gives the constitutive relation

$$
\mathbf{B}=\mu_{0}(\mathbf{H}+\mathbf{M})=\mu_{0} \mu_{r} \mathbf{H}+\mu_{0} \mathbf{M}_{0}
$$

where $\mu_{r}$ is the relative permeability. The 3-D magnetic vector potential can be expression in the form of the Poisson equation

$$
\nabla^{2} \mathbf{A}=-\mu_{0}\left(\nabla \times \mathbf{M}_{0}\right)-\mu \mathbf{J}
$$

where $\mathbf{J}$ is the current density distribution of the coils. Both the current density distribution [5] and the magnetization vector [7], [8] can be modeled in a 3-D Euclidian space with a double Fourier series. For example, a rectangular current in the $x$-direction, $J_{x}$, is described by

$$
\begin{aligned}
J_{x}= & J_{x_{c c}} \cos \left(\omega_{k} x\right) \cos \left(\omega_{l} y\right)+J_{x_{c s}} \cos \left(\omega_{k} x\right) \sin \left(\omega_{l} y\right) \\
& +J_{x_{s c}} \sin \left(\omega_{k} x\right) \cos \left(\omega_{l} y\right)+J_{x_{s s}} \sin \left(\omega_{k} x\right) \sin \left(\omega_{l} y\right)
\end{aligned}
$$

with spatial frequencies

$$
\begin{aligned}
& \omega_{k}=\frac{k \pi}{\tau_{x}} \\
& \omega_{l}=\frac{l \pi}{\tau_{y}} .
\end{aligned}
$$

It is assumed that the current is only flowing in the tangential, $x, y$-direction, and, that the magnetization vector is symmetrical in the $x, y$ plane. In combination with the Coulomb Gauge condition, $\nabla \cdot \mathbf{A}=0$, the following solution for the magnetic vector can be obtained

$$
\begin{aligned}
& A_{x}=\sum_{k=0}^{\infty} \sum_{l=0}^{\infty} \\
& \quad\left[\frac{\omega_{l}}{\lambda}\left(c_{1} e^{\lambda z}+c_{2} e^{-\lambda z}\right)+C_{x_{c s}}\right) \cos \left(\omega_{k} x\right) \sin \left(\omega_{l} y\right) \\
& \quad+\left(\frac{\omega_{l}}{\lambda}\left(c_{3} e^{\lambda z}+c_{4} e^{-\lambda z}\right)+C_{x_{s c}}\right) \sin \left(\omega_{k} x\right) \cos \left(\omega_{l} y\right) \\
& +\left(\frac{\omega_{l}}{\lambda}\left(c_{5} e^{\lambda z}+c_{6} e^{-\lambda z}\right)+C_{x_{c c}}\right) \cos \left(\omega_{k} x\right) \cos \left(\omega_{l} y\right) \\
& \left.\quad+\left(\frac{\omega_{l}}{\lambda}\left(c_{7} e^{\lambda z}+c_{8} e^{-\lambda z}\right)+C_{x_{s s}}\right) \sin \left(\omega_{k} x\right) \sin \left(\omega_{l} y\right)\right] \\
& \left.A_{y}=\sum_{k=0}^{\infty} \sum_{l=0}^{\infty}\right) \\
& \left.\quad+\frac{-\omega_{k}}{\lambda}\left(c_{1} e^{\lambda z}+c_{2} e^{-\lambda z}\right)+C_{y_{s c}}\right) \sin \left(\omega_{k} x\right) \cos \left(\omega_{l} y\right) \\
& \quad+\left(\frac{-\omega_{k}}{\lambda}\left(c_{3} e^{\lambda z}+c_{4} e^{-\lambda z}\right)+C_{y_{c s}}\right) \cos \left(\omega_{k} x\right) \sin \left(\omega_{l} y\right) \\
& +\left(\frac{\omega_{k}}{\lambda}\left(c_{5} e^{\lambda z}+c_{6} e^{-\lambda z}\right)+C_{y_{s s}}\right) \sin \left(\omega_{k} x\right) \sin \left(\omega_{l} y\right) \\
& \left.+\left(\frac{\omega_{k}}{\lambda}\left(c_{7} e^{\lambda z}+c_{8} e^{-\lambda z}\right)+C_{y_{c c}}\right) \cos \left(\omega_{k} x\right) \cos \left(\omega_{l} y\right)\right] \\
& A_{z}=0
\end{aligned}
$$

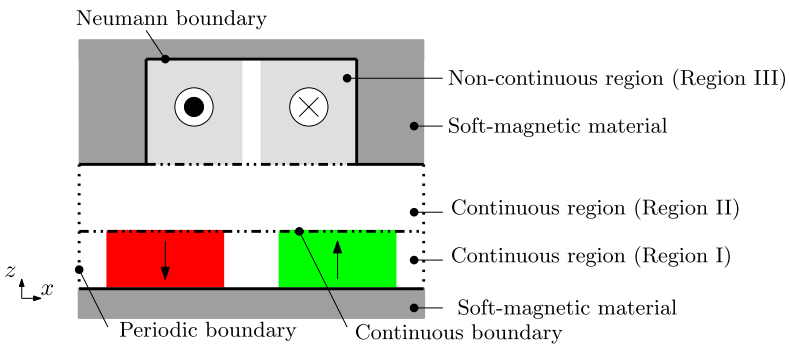

Fig. 1. Part of a linear motor, including the division in regions and boundary conditions.

where

$$
\lambda=\sqrt{\omega_{k}^{2}+\omega_{l}^{2}} .
$$

The constants $C$ are related to the magnetization vector and the current density distribution and are equal to

$$
\begin{aligned}
C_{x_{c s}} & =\frac{\mu_{0} \omega_{l} M_{z_{c c}}-\mu J_{x_{c s}}}{\omega_{k}^{2}+\omega_{l}^{2}} \\
C_{x_{s c}} & =\frac{-\mu_{0} \omega_{l} M_{z_{s s}}-\mu J_{x_{s c}}}{\omega_{k}^{2}+\omega_{l}^{2}} \\
C_{x_{c c}} & =\frac{-\mu_{0} \omega_{l} M_{z_{c s}}-\mu J_{x_{c c}}}{\omega_{k}^{2}+\omega_{l}^{2}} \\
C_{x_{s s}} & =\frac{\mu_{0} \omega_{l} M_{z_{s c}}-\mu J_{x_{s s}}}{\omega_{k}^{2}+\omega_{l}^{2}} \\
C_{y_{s c}} & =\frac{-\mu_{0} \omega_{k} M_{z_{c c}}-\mu J_{y_{s c}}}{\omega_{k}^{2}+\omega_{l}^{2}} \\
C_{y_{c s}} & =\frac{\mu_{0} \omega_{k} M_{z_{s s}}-\mu J_{y_{c s}}}{\omega_{k}^{2}+\omega_{l}^{2}} \\
C_{y_{s s}} & =\frac{-\mu_{0} \omega_{k} M_{z_{c s}}-\mu J_{y_{s s}}}{\omega_{k}^{2}+\omega_{l}^{2}} \\
C_{y_{c c}} & =\frac{\mu_{0} \omega_{k} M_{z_{s c}}-\mu J_{y_{c c}}}{\omega_{k}^{2}+\omega_{l}^{2}} .
\end{aligned}
$$

To derive a solution for the magnetic fields by means of the harmonic model, an expression of the magnetic vector potential is obtained for each region. The unknown coefficients, $c_{1}-c_{8}$, are solved by applying boundary conditions between the different regions. The continuous boundary conditions are applied between regions with an identical pitch, in which the magnetizing vector is embedded in the tangential components, as defined in (4). At the boundary of a region with soft-magnetic material or at the border of a region with infinite height the Dirichlet and Neumann boundary condition appear, respectively. Inside non-continuous regions, the Neumann boundary condition must yield at the vertical boundaries. Therefore, these regions have a pitch equal to twice the length of the region [9]. Continuous regions have a periodical boundary conditions at both vertical boundaries of the geometry. This boundary condition is fulfilled by describing the $x$ - and $y$-component as a double Fourier series. Finally, at the boundary between a continuous and a noncontinuous region, a combination of continuous and Neumann boundary conditions needs to be applied, as shown in Fig. 1. Since the two neighboring regions have a different spatial frequency, mode-matching needs to be applied [4]. 
The mode-matching technique is based on the fact that magnetic fields, described as Fourier series, are identical at both sides of the boundary. This means that the coefficients of the Fourier series in one region can be expressed as a function of the periodicity of the Fourier series in the other region and vice versa, as illustrated in [9]. Along the entire boundary of the continuous region, a boundary condition is defined for the tangential components, as shown in Fig. 1. Therefore, these boundary conditions are evaluated as a function of the harmonics in the continuous region. The boundary condition for the normal component of the magnetic fields is only defined across the overlapping area of both regions, and therefore, it is solved as a function of the harmonics in the non-continuous region.

The 3-D harmonic modeling cannot only be applied in motors [8], [10], but is also able to model contactless energy transfer systems by means of an inductive coupling [5]. The model is able to take different kind of boundaries around the coils into account, such as soft-magnetic plates and complex structures with cavities with or without a coil inside. The 3-D model can be simplified to a 2-D one, by assuming an infinite length in the $x$ - or $y$-direction. Thereby, the harmonic $k$ or $l$ becomes equal to zero, resulting in an expression for the magnetic fields with a single Fourier series.

\section{B. Charge Modeling}

For non-periodical actuator or motor structures, charge modeling can be applied as an alternative to the harmonic model. In charge models, every PM is modeled individually. The charge model can be derived from the scalar potential $\Psi$, that is

$$
\begin{aligned}
\mathbf{H} & =-\nabla \Psi \\
\nabla^{2} \Psi & =\nabla \cdot \mathbf{M} .
\end{aligned}
$$

For a magnet with a uniform magnetization, the scalar potential is given by

$$
\Psi=\frac{1}{4 \pi} \oint_{s} \frac{\mathbf{M}\left(\mathbf{x}^{\prime}\right) \cdot \mathbf{n}}{\left|\mathbf{x}-\mathbf{x}^{\prime}\right|} \mathrm{d} S
$$

where $\mathbf{n}$ is the normal to the magnet surface $S$. For a magnet with a parallel magnetization in the positive $z$-direction, the model results in a surface charge model, as shown in Fig. 2, with two surface charges $\sigma=\mathbf{M} \cdot \mathbf{n}$. The magnetic flux density is described by [6]

$$
\begin{aligned}
& B_{x}=\frac{B_{r}}{4 \pi} \sum_{i=0}^{1} \sum_{j=0}^{1} \sum_{k=0}^{1}(-1)^{i+j+k} \log (R-T) \\
& B_{y}=\frac{B_{r}}{4 \pi} \sum_{i=0}^{1} \sum_{j=0}^{1} \sum_{k=0}^{1}(-1)^{i+j+k} \log (R-S) \\
& B_{z}=\frac{B_{r}}{4 \pi} \sum_{i=0}^{1} \sum_{j=0}^{1} \sum_{k=0}^{1}(-1)^{i+j+k} \tan ^{-1}\left(\frac{S T}{R U}\right)
\end{aligned}
$$

where

$$
\begin{aligned}
R & =\sqrt{S^{2}+T^{2}+U^{2}} \\
S & =x-(-1)^{i} l_{m} / 2
\end{aligned}
$$

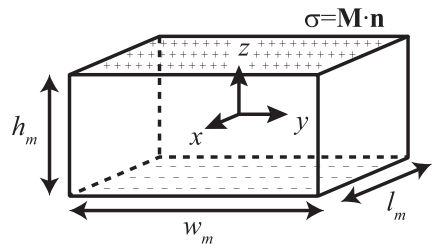

Fig. 2. Charge model of a magnet which has a parallel magnetization in the positive $z$-direction.

$$
\begin{aligned}
T & =y-(-1)^{j} w_{m} / 2 \\
U & =z-(-1)^{k} h_{m} / 2 .
\end{aligned}
$$

this method assumes that the permeability in the whole domain is equal to unity. Magnetic materials can be included by the method of imaging. In addition, the relative permeability, $\mu_{r}$, of a magnet array, such as applied in planar motors can be considered in this way by mirroring $n$-times in both the top and bottom side of the magnet array itself [11]. The magnetization of each layer of images is equal to

$$
\mathbf{M}_{n}=\frac{2}{\mu_{r}+1}\left(-\frac{\mu_{r}-1}{\mu_{r}+1}\right)^{n} \mathbf{M}_{0} .
$$

Due to the low-relative permeability of modern NdFeB magnets $\left(\mu_{r}=1.03-1.05\right)$, the adjustment of the magnetization of the magnets itself $(n=0)$ is sufficient to reduce the error of the magnetic flux density prediction with a factor 10 [11].

\section{LINEAR MOTORS}

Linear PM motors are widely used as direct-drive solutions in the industry because they offer good servo performance and high-force densities. In high-precision applications, the structure of such motors usually adopts an air-cored coil assembly, which is positioned inside a U-shaped stator with a double PM array. Owing to a constant reluctance path for the PM field through the moving coil assembly, the coreless structure does not suffer from end-effects or cogging forces. As a result, this topology merits itself in terms of low-force ripples and, hence, a good force predictability. Iron-cored linear motors offer higher force densities compared with their coreless counterpart by embedding the coils inside a laminated yoke with slotting. However, they intrinsically suffer from the aforementioned force ripples, which should be minimized when it is desirable to exploit the high-force capabilities of iron-cored motors in high-precision applications.

Ripple reduction by means of a design optimization, requires a fast and precise modeling technique, which includes both the slotting and finite length of the yoke. Although both effects can be considered with magnetic equivalent circuit modeling [12], [13], the circuit continuously changes when the slotted yoke is moved with respect to the PM array. In addition, the coarse discretization of the magnetic structure and the difficult nature of the airgap permeances result in an inaccurate prediction of the force ripples. A more accurate method of predicting the 2-D magnetic field distribution, and, hence, the force ripples is achieved by means of a conformal mapping [14]. In this method, the complex geometrical structure of the linear motor is mapped to a rectangular 


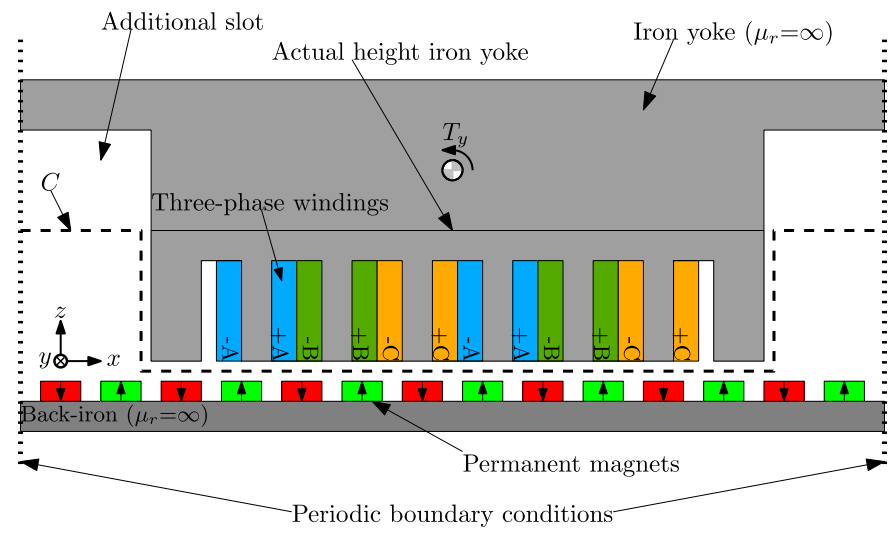

Fig. 3. Schematic view of the linear PM motor, including the extra slot for modeling the finite yoke length.

shaped domain with a complex mapping function. Inside the rectangular domain, the magnetic field distribution is more easily calculated. Because it is impossible to find an analytical expression for the complex mapping functions when many slots are considered, this method has to be used in conjunction with numerical toolboxes, which are computationally demanding and do not always guarantee convergence.

A direct expression for the 2-D magnetic flux distribution can also be obtained from the harmonic model presented in Section II. Aside from describing the fields in the airgap and PM array alone, this method also makes it possible to obtain the fields inside the different slots when each of them is considered as a separate region. As an example, the fields are calculated in a commercially available linear motor. The model of the motor is shown in Fig. 3. The PM array adopts a north-south magnetization and has a pole pitch of $12 \mathrm{~mm}$. The coil unit comprises six concentrated coils and a laminated iron yoke. The clearance between the yoke and magnet array is equal to $1 \mathrm{~mm}$. To account for the finite yoke length inside the analytical model, the structure, shown in Fig. 3, includes an extra slot, which has a larger width and height compared with the other slots. The inclusion of the extra slot, however, results in an increased yoke height. It is important to note that the width of the extra slot is chosen such that the modeled domain inhabits periodicity. In the considered problem, the magnetic fields in 10 regions are calculated.

Provided the flux density distribution, the force vector acting on the coils and yoke can be calculated by means of the Maxwell stress tensor

$$
\begin{aligned}
& \mathbf{F}=\frac{1}{\mu} \oint_{S} \mathbb{T} \cdot \mathbf{n} d S \\
& \mathbf{T}=\frac{1}{\mu} \oint_{S} \mathbf{r} \times \mathbb{T} \cdot \mathbf{n} d S
\end{aligned}
$$

where $S$ is a closed surface surrounding the body, $\mathbf{n}$ is the outward normal vector to $S$, and $\mathbf{r}$ is the arm to the point of rotation. Usually, this point is the center point of mass of the moving member. The Maxwell stress tensor, $\mathbb{T}$, is given by

$$
\mathbb{T}=\left[\begin{array}{ccc}
\left(B_{x}^{2}-\frac{1}{2}|\vec{B}|^{2}\right) & B_{x} B_{y} & B_{x} B_{z} \\
B_{y} B_{x} & \left(B_{y}^{2}-\frac{1}{2}|\vec{B}|^{2}\right) & B_{y} B_{z} \\
B_{z} B_{x} & B_{z} B_{y} & \left(B_{z}^{2}-\frac{1}{2}|\vec{B}|^{2}\right)
\end{array}\right] .
$$

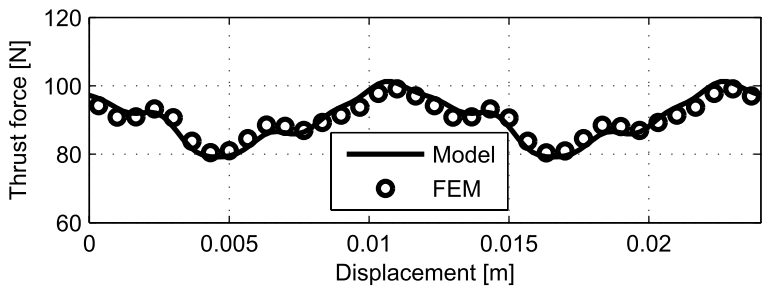

(a)

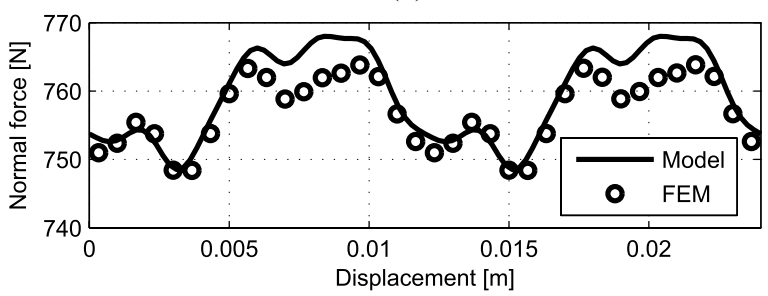

(b)

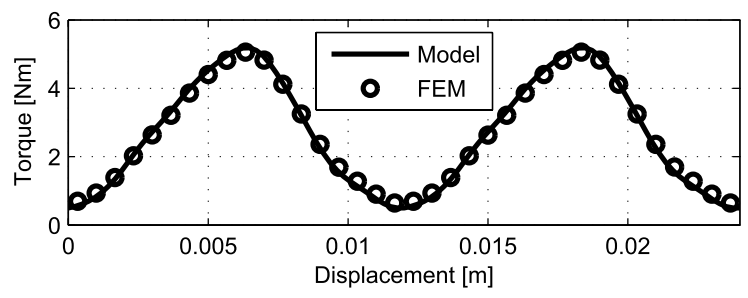

(c)

Fig. 4. (a) Thrust force, (b) normal force, and (c) torque predicted with the analytical model and FE-model.

In (32), the integral is evaluated along a line through the airgap. Fig. 4 shows the thrust and normal force when the motor is moved over two pole pitches with respect to the PM array. The three-phase currents are commutated such as to produce maximum thrust force for a peak current density of $8 \mathrm{~A} \mathrm{~mm}^{-2}$. Fig. 4(a) and (b) also shows the results obtained from FEA and it is concluded that the thrust and normal force predicted with the analytical model have a maximum error of $1 \%$.

In some applications, it is also desirable to know the torque acting on the linear motor [15], [16]. The torque is calculated with (33), but in this case the integral has to be evaluated along a contour, which closely follows the outer shape of the yoke [17]. This contour is shown in Fig. 3. As shown in Fig. 4(c), the calculated torque shows good agreement with FEA.

\section{Planar Motors}

Planar motors or actuators are capable of delivering motion in a horizontal plane. Several types of planar motors can be distinguished based on the number of DOF and the range of motion of the DOF. For high-precision applications, 6 DOF, long-stroke planar motors, which can both provide magnetic levitation and propulsion in the $x y$-plane have been investigated in the past decades [18]-[23]. These motors can either be constructed with stationary magnets and moving coils or the other way around with moving magnets and stationary coils. Moving-magnet motors are usually coreless. In moving-coil motors iron could be used behind the stationary PM array. 


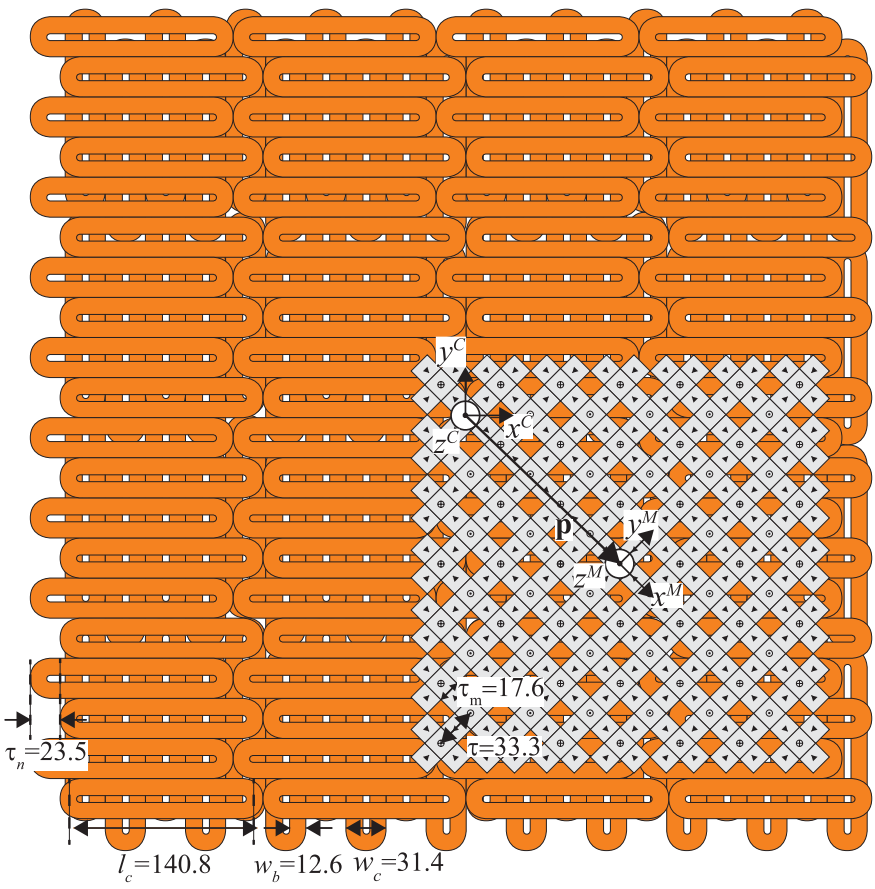

(a)

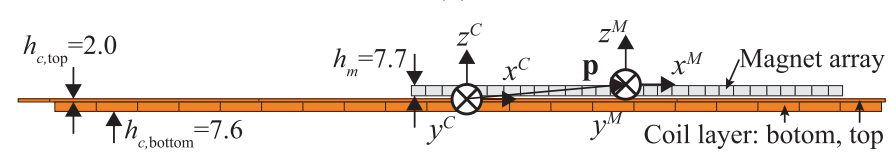

(b)

Fig. 5. Double-layer planar motor [21]. (a) Top view. (b) Side view. Dimensions in millimeter.

An example of a 6 DOF moving-magnet planar motors is shown in Fig. 5 [21]. The translator contains $281 \mathrm{PM}$ arranged in a quasi-Halbach magnetization. The coil array (160 coils) consists of two layers of coils. The top layer (horizontally oriented) can produce a levitation force and a force in the $y$-direction, whereas the bottom layer (vertically oriented) can produce a levitation force and a force in the $x$-direction. As the magnetic flux density of the coils reduces exponentially with the distance from the magnet surface, the height of the bottom layer $(7.6 \mathrm{~mm})$ is larger than the height of the top layer $(2.0 \mathrm{~mm})$ to distribute the power dissipation equally over the coils. Each coil is connected to its own power amplifier.

To predict the force and torque in planar motors, finite element methods are not suitable as planar motors have a large volume in which the energy conversion takes place and which requires a dense mesh. Therefore, analytical techniques are used for the analysis and design of these motors. As the forces in ironless planar motors are only based on the interaction between the PM array and the current carrying coils, the force and torque exerted on the magnets are calculated with the Lorentz force law

$$
\begin{aligned}
& \mathbf{F}=-\iiint_{V_{\text {coil }}} \mathbf{J} \times \mathbf{B} \mathrm{d} V \\
& \mathbf{T}=-\iiint_{V_{\text {coil }}} \mathbf{r} \times \mathbf{J} \times \mathbf{B} \mathrm{d} V .
\end{aligned}
$$

\section{A. Harmonic Model}

The magnetic flux density of a planar magnet array can be effectively modeled with a harmonic model [7], [8]. An accurate expression can be obtained for the magnetic flux density of the full magnet array with a low number of harmonics at the consequence that the end-effects are not included. Therefore, harmonic models are most suitable for the modeling of moving-coil planar motors. The magnetic flux density of the magnet array with quasi-Halbach magnetization, as shown in Fig. 5, can be described in the region of the coils as

$$
\mathbf{B}=\sum_{k=1}^{\infty} \sum_{l=1}^{\infty} K e^{\lambda z^{M}}\left[\begin{array}{c}
\omega_{k} \sin \left(\omega_{k} x^{M}\right) \cos \left(\omega_{l} y^{M}\right) \\
\omega_{l} \cos \left(\omega_{k} x^{M}\right) \sin \left(\omega_{l} y^{M}\right) \\
-\lambda \cos \left(\omega_{k} x^{M}\right) \cos \left(\omega_{l} y^{M}\right)
\end{array}\right]
$$

where

$$
\begin{aligned}
K= & \frac{B_{r} e^{\lambda\left(-m_{b}\right)}\left(e^{\lambda m_{b}}-e^{\lambda m_{t}}\right)}{\pi \lambda\left(k^{2}+l^{2}\right)\left(\left(\mu_{r}-1\right)^{2} e^{2 \lambda m_{b}}-\left(\mu_{r}+1\right)^{2} e^{2 \lambda m_{t}}\right)} \\
& \left(( \mu _ { r } - 1 ) e ^ { \lambda m _ { b } } \left(a(k)\left(\pi a(l)\left(k^{2}+l^{2}\right)-\lambda l \tau b(l)\right)\right.\right. \\
& -\lambda k \tau a(l) b(k))-\left(\mu_{r}+1\right) e^{\lambda m_{t}}(a(k)(\pi a(l) \\
& \left.\left.\left.\left(k^{2}+l^{2}\right)+\lambda l \tau b(l)\right)+\lambda k \tau a(l) b(k)\right)\right) \\
a(k)= & \frac{4}{k \pi} \cos \left(\frac{k\left(\tau-\tau_{m}\right) \pi}{2 \tau}\right) \sin \left(\frac{k \pi}{\tau}\right) \\
b(k)= & \frac{4}{k \pi} \sin \left(\frac{k\left(\tau-\tau_{m}\right) \pi}{2 \tau}\right) \sin \left(\frac{k \pi}{\tau}\right)
\end{aligned}
$$

and where $\omega_{x}=\omega_{y}, \tau$ is the pole pitch, $\tau_{m}$ is the size of the magnet magnetized in the $x$-direction, and $m_{b}$ and $m_{t}$ are the $z$-coordinates of the bottom and top of the magnet array, respectively. It should be noted that also in this method the air holes in the magnet array are assumed to have the same permeability as the magnets.

The Lorentz force and torque integrals over the coils can be obtained analytically in the $z$-direction, and the integrals in the $x$ - and $y$-directions can be solved analytically for straight current carrying volumes parallel or at an angle of $45^{\circ}$ with respect to the magnet array. As a result, the harmonic modeling technique gives fast calculation results for these coil orientations [8].

\section{B. Charge Model}

With the charge model, every individual magnet in the magnet array can be modeled and, hence, end-effects and variations in the magnet array, due to, e.g., manufacturing tolerances, can be included [24]. Furthermore, the force on the individual magnets, and therefore, the force distribution inside a planar magnet array can be predicted [25]. The Lorentz force and torque have to be calculated numerically. Only an analytical expression can be obtained when a straight current carrying volume is parallel to the sides of a magnet [26].

The force distribution in the magnet array will mechanical deform it. Two causes of deformation can be distinguished. Deformations due to the forces exerted by the current carrying coils, which can be obtained by calculating the force on each magnet using the charge method and static deformations due to the forces between the individual magnets. 


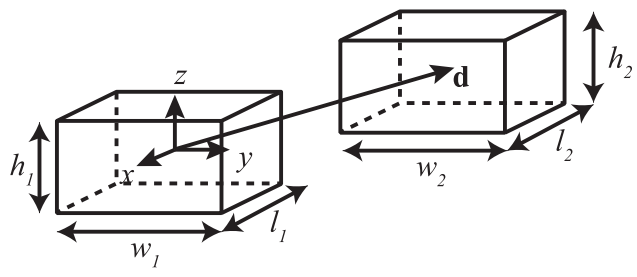

Fig. 6. Dimensions of two cuboidal magnets.

The static force between two magnets (dimensions defined in Fig. 6) with parallel magnetization in the $z$-direction is given by [6]

$$
\mathbf{F}=\sum_{i=0}^{1} \sum_{j=0}^{1} \sum_{k=0}^{1} \sum_{l=0}^{1} \sum_{p=0}^{1} \sum_{q=0}^{1}(-1)^{i+j+k+l+p+q} \psi
$$

where

$$
\begin{aligned}
\psi_{x}= & \left.\frac{1}{2}\left(T^{2}-U^{2}\right) \log (R-S)+S T \log (R-T)\right) \\
& +T U \tan ^{-1}\left(\frac{S T}{R U}\right)+\frac{1}{2} R S \\
\psi_{y}= & \left.\frac{1}{2}\left(S^{2}-U^{2}\right) \log (R-T)+S T \log (R-S)\right) \\
& +S U \tan ^{-1}\left(\frac{S T}{R U}\right)+\frac{1}{2} R T \\
\psi_{z}= & -S U \log (R-S)-T U \log (R-T) \\
& +S T \tan ^{-1}\left(\frac{S T}{R U}\right)-R U \\
S= & d_{x}-(-1)^{i} \frac{l_{1}}{2}+(-1)^{j} \frac{l_{2}}{2} \\
T= & d_{y}-(-1)^{k} \frac{w_{1}}{2}+(-1)^{l} \frac{w_{2}}{2} \\
U= & d_{z}-(-1)^{p} \frac{h_{1}}{2}+(-1)^{q} \frac{h_{2}}{2} \\
R= & \sqrt{S^{2}+T^{2}+U^{2}} .
\end{aligned}
$$

The expression of the force between two magnets with perpendicular magnetization vectors is given in [27].

When the forces on the individual magnet are known, the displacement of the magnet assembly can be determined from a mechanical model. A mechanical FEA model can be used to extract the mode shapes $\Omega$ and eigen frequencies $\Phi$ of the magnet assembly. The quasi-static deformation $\Delta \mathbf{p}(t)$ due to a force distribution $\mathbf{F}(t)$ is described by

$$
\Delta \mathbf{p}(t)=\Phi\left(\Omega^{2}\right)^{-1} \Phi^{\top} \mathbf{F}(t) .
$$

The amplitude of each mode shape $A_{n}$ is given by

$$
A_{n}=\Phi_{n} \mathbf{F}
$$

where $\Phi_{n}$ is the column of $\Phi$ corresponding to mode shape $n$.

\section{Real-Time Control}

To control a planar motor, decoupling algorithms for the force and torque are applied [28]. For that purpose, at every sample time the relation between the coil currents and the

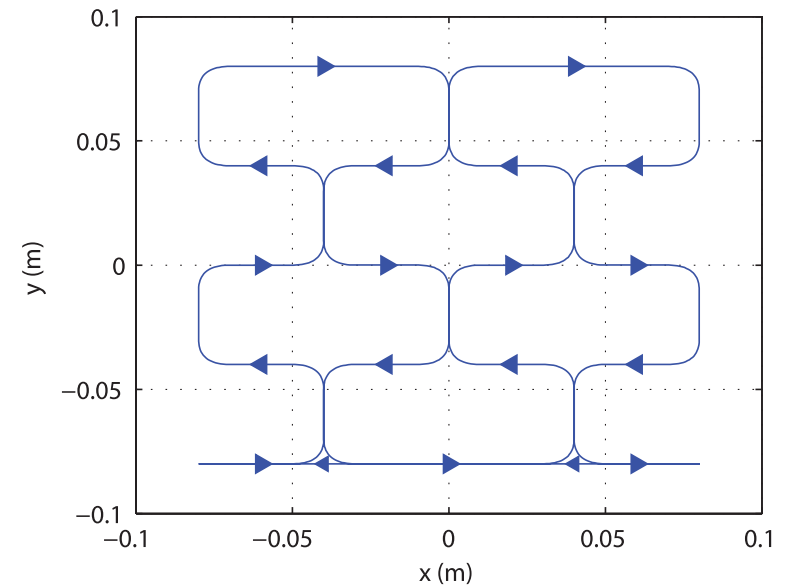

Fig. 7. Typical trajectory of a planar motor in the $x y$-plane.

force, torque and amplitude of the mode shapes of the translator should be obtained. They can be determined from the presented analytical models. As planar motors are inherently linear, superposition can be applied, and the total transfer function for a system with $N_{c}$ coils may be described as $\mathbf{w}=\Gamma(\mathbf{p}) \mathbf{i}$

$$
\Gamma(\mathbf{p})=\left[\begin{array}{cccc}
F_{x, 1} & F_{x, 2} & \cdots & F_{x, N_{c}} \\
F_{y, 1} & F_{y, 2} & \cdots & F_{y, N_{c}} \\
F_{z, 1} & F_{z, 2} & \cdots & F_{z, N_{c}} \\
T_{x, 1} & T_{x, 2} & \cdots & T_{x, N_{c}} \\
T_{y, 1} & T_{y, 2} & \cdots & T_{y, N_{c}} \\
T_{z, 1} & T_{z, 2} & \cdots & T_{z, N_{c}} \\
A_{1,1} & A_{1,2} & \cdots & A_{1, N_{c}} \\
A_{2,1} & A_{2,2} & \cdots & A_{2, N_{c}} \\
\vdots & \vdots & \ddots & \vdots \\
A_{n, 1} & A_{n, 2} & \cdots & A_{n, N_{c}}
\end{array}\right]
$$

where $\mathbf{p}$ its the position of the translator and $\mathbf{w}$ is the wrench vector containing the total force, torque, and mode shapes

$$
\mathbf{w}=\left[\begin{array}{llllllllll}
F_{x} & F_{y} & F_{z} & T_{x} & T_{y} & T_{z} & A_{1} & A_{2} & \cdots & A_{n}
\end{array}\right]^{\top} .
$$

Each element of $\Gamma$ describes the interaction between a coil and the force, torque or the mode-shape of the translator per unit of current. To control the planar motor in real-time, the current should be obtained from the inverse of the mapping $\Gamma$. The coil currents $\mathbf{i}$ to obtain a certain wrench vector $\mathbf{w}$ is equal to

$$
\mathbf{i}=\Gamma(\mathbf{p})^{-1} \mathbf{w}=\Gamma(\mathbf{p})^{\top}\left(\Gamma(\mathbf{p}) \Gamma(\mathbf{p})^{\top}\right)^{-1} \mathbf{w} .
$$

If the force and torque model are based on the harmonic model, real-time analytical expressions for the mapping can be derived (while considering only the first harmonic) [8], [29]. In other cases, for example when the forces and torques are based on the surface charge model, also look-up tables can be applied to determine the mapping at each position.

In Fig. 7, a typical trajectory in the $x y$-plane of a planar motor is shown. Fig. 8 shows the corresponding acceleration profile and the deformation of the translator of the planar motor. In this simulation, the mode shapes have not been controlled by the commutation algorithm. It can be observed that largest deformation is in the first mode shape $\left(A_{1, \mathrm{rms}}=\right.$ $267 \mathrm{~nm}$ ), whereas the other mode shapes have at least a factor 

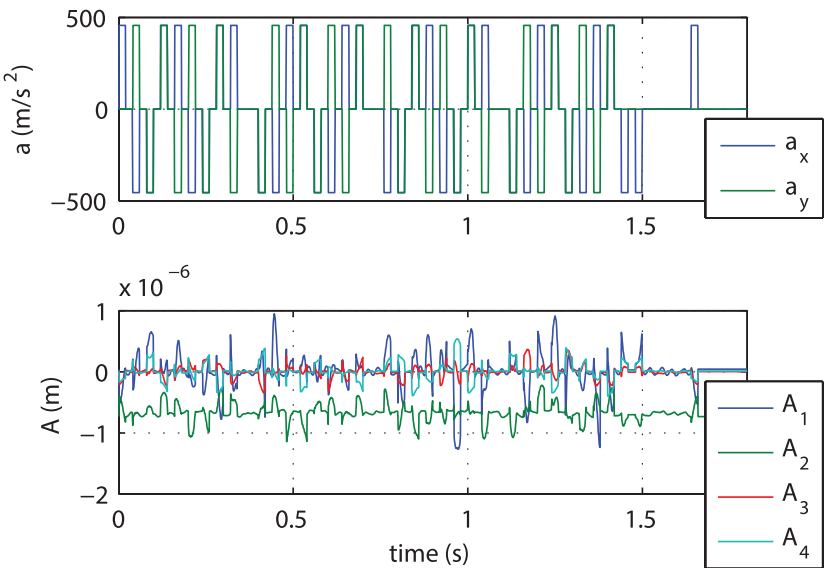

Fig. 8. Acceleration profile and mode shape amplitudes during levitation and fast motion in the $x y$-plane over the trajectory in Fig. 7.

two smaller amplitude. The static deformation of the magnet assembly is clearly visible in the second mode shape $A_{2}$. Obtaining these results from FEA is impossible as the force calculation algorithms require that all magnets are surrounded by an air layer. Given typical gaps of 30-50 $\mu \mathrm{m}$ between magnets, this would require a too large mesh. Therefore, analytical models are indispensable for the design of planar motors.

\section{CONCLUSION}

An overview of analytical techniques for the design of linear and planar motors is given. These methods can be used in parallel or as an alternative to FEA. Harmonic modeling is the most suitable method for 2-D analysis of slotted and slotless motors and can in certain situations also be applied to 3-D structures. Models based on magnetic surface charges are essential for the design of magnetically levitated planar motors and coreless linear motors as they not only can predict the force and torque on the total magnet assembly, but also their distributions. Furthermore, individual variation of magnet properties and dimensions can be considered.

\section{REFERENCES}

[1] N. Boules, "Two-dimensional field analysis of cylindrical machines with permanent magnet excitation," IEEE Trans. Ind. Appl., vol. IA-20, no. 5, pp. 1267-1277, Sep. 1984.

[2] Z. Zhu, D. Howe, E. Bolte, and B. Ackermann, "Instantaneous magnetic field distribution in brushless permanent magnet dc motors. I. Opencircuit field," IEEE Trans. Magn., vol. 29, no. 1, pp. 5698-5708, Jan. 1993.

[3] J. Wang, D. Howe, and G. W. Jewell, "Fringing in tubular permanentmagnet machines: Part I. Magnetic field distribution, flux linkage, and thrust force," IEEE Trans. Magn., vol. 39, no. 6, pp. 3507-3516, Nov. 2003.

[4] B. L. J. Gysen, K. J. Meessen, J. J. H. Paulides, and E. A. Lomonova, "General formulation of the electromagnetic field distribution in machines and devices using fourier analysis," IEEE Trans. Magn., vol. 45 , no. 6, pp. 39-52, Jan. 2010.

[5] J. P. C. Smeets, T. T. Overboom, J. W. Jansen, and E. A. Lomonova, "Three-dimensional analytical modeling technique of electromagnetic fields of air-cored coils surrounded by different ferromagnetic boundaries," IEEE Trans. Magn., vol. 49, no. 12, pp. 5698-5708, Dec. 2013.

[6] G. Akoun and J.-P. Yonnet, "3D analytical calculation of the forces exerted between two cuboidal magnets," IEEE Trans. Magn., vol. 20, no. 5, pp. 1962-1964, Sep. 1984

[7] H.-S. Cho, C.-H. Im, and H.-K. Jung, "Magnetic field analysis of 2-D permanent magnet array for planar motor," IEEE Trans. Magn., vol. 37, no. 56, pp. 3762-3766, Sep. 2001.
[8] J. W. Jansen, C. M. M. van Lierop, E. A. Lomonova, and A. J. A. Vandenput, "Modeling of magnetically levitated planar actuators with moving magnets," IEEE Trans. Magn., vol. 43, no. 1, pp. 15-25, Jan. 2007.

[9] J. P. C. Smeets, T. T. Overboom, J. W. Jansen, and E. A. Lomonova, "Mode-matching technique applied to three-dimensional magnetic field modeling," IEEE Trans. Magn., vol. 48, no. 11, pp. 3383-3386, Nov. 2012.

[10] K. J. Meessen, J. J. H. Paulides, and E. A. Lomonova, "Force calculations in 3-D cylindrical structures using Fourier analysis and the Maxwell stress tensor," IEEE Trans. Magn., vol. 49, no. 1, pp. 536-545, Jan. 2013

[11] J. M. M. Rovers, J. W. Jansen, and E. A. Lomonova, "Modeling of relative permeability of permanent magnet material using magnetic surface charges," IEEE Trans. Magn., vol. 49, no. 6, pp. 2913-2919, Jun. 2013.

[12] H. Polinder, J. G. Slootweg, M. J. Hoeijmakers, and J. C. Compter, "Modeling of a linear pm machine including magnetic saturation and end effects: Maximum force-to-current ratio," IEEE Trans. Ind. Appl., vol. 39, no. 6, pp. 1681-1688, Nov./Dec. 2003.

[13] B. Sheikh-Ghalavand, S. Vaez-Zadeh, and A. H. Isfahani, "An improved magnetic equivalent circuit model for iron-cored linear permanentmagnet synchronous motors," IEEE Trans. Magn., vol. 46, no. 1, pp. 112-120, Jan. 2010.

[14] D. C. J. Krop, E. A. Lomonova, and A. J. A. Vandenput, "Application of Schwarz-Christoffel mapping to permanent-magnet linear motor analysis," IEEE Trans. Magn., vol. 44, no. 3, pp. 352-359, Mar. 2008.

[15] T. T. Overboom, J. P. C. Smeets, J. W. Jansen, and E. A. Lomonova "Topology comparison for a magnetically suspended ceiling actuator," in Proc. IEEE IEMDC, May 2011, pp. 296-301.

[16] G. Rothenhofer and A. Slocum, "Reducing pitch error of a linear motion system actuated by a permanent magnet open face linear motor," Precis. Eng., vol. 33, no. 3, pp. 305-309, Jul. 2009.

[17] T. T. Overboom, J. P. C. Smeets, J. W. Jansen, and E. A. Lomonova, "Semianalytical calculation of the torque in a linear permanent-magnet motor with finite yoke length," IEEE Trans. Magn., vol. 48, no. 11, pp. 3575-3578, Nov. 2012.

[18] J. C. Compter, "Electro-dynamic planar motor," Precis. Eng., vol. 28, no. 2, pp. 171-180, Apr. 2004.

[19] W. J. Kim and D. L. Trumper, "High-precision magnetic levitation stage for photolithography," Precis. Eng., vol. 22, no. 2, pp. 66-77, Apr. 1998.

[20] J. W. Jansen, C. M. M. van Lierop, E. A. Lomonova, and A. J. A. Vandenput, "Magnetically levitated planar actuator with moving magnets," IEEE Trans. Ind. Appl., vol. 44, no. 4, pp. 1108-1115, Jul. 2008.

[21] J. M. M. Rovers, J. W. Jansen, and E. A. Lomonova, "Design and measurements of the double layer planar motor," in Proc. IEEE IEMDC, May 2013, pp. 204-211.

[22] Y. Ueda and H. Ohsaki, "A planar actuator with a small mover traveling over large yaw and translational displacements," IEEE Trans. Magn., vol. 44, no. 5, pp. 609-616, May 2008.

[23] J. de Boeij, E. Lomonova, and J. Duarte, "Contactless planar actuator with manipulator: A motion system without cables and physical contact between the mover and the fixed world," IEEE Trans. Ind. Appl., vol. 45, no. 6, pp. 1930-1938, Nov. 2009.

[24] J. M. M. Rovers, J. W. Jansen, and E. A. Lomonova, "Force and torque errors due to manufacturing tolerances in planar actuators," IEEE Trans. Magn., vol. 48, no. 11, pp. 3116-3119, Nov. 2012.

[25] J. M. M. Rovers, J. W. Jansen, J. C. Compter, and E. A. Lomonova, "Analysis method of the dynamic force and torque distribution in the magnet array of a commutated magnetically levitated planar actuator," IEEE Trans. Ind. Electron., vol. 59, no. 5, pp. 2157-2166, May 2012.

[26] J. M. M. Rovers, J. W. Jansen, and E. A. Lomonova, "Analytical calculation of the force between a rectangular coil and a cuboidal permanent magnet," IEEE Trans. Magn., vol. 46, no. 6, pp. 1656-1659, Jun. 2010.

[27] J. L. G. Janssen, J. J. H. Paulides, E. A. Lomonova, F. Bölöni, A. Tounzi, and F. Piriou, "Analytical calculation of interaction force between orthogonally magnetized permanent magnets," Sensor Lett., vol. 7, no. 3, pp. 442-445, 2009.

[28] C. M. M. van Lierop et al., "Commutation of a magnetically levitated planar actuator with moving-magnets," IEEJ Trans. Ind. Appl., vol. 128 , no. 12 , pp. 1333-1338, 2008.

[29] J. Peng, Y. Zhou, and G. Liu, "Calculation of a new real-time control model for the magnetically levitated ironless planar motor," IEEE Trans. Magn., vol. 49, no. 4, pp. 1416-1422, Apr. 2013. 